\title{
Malaria incidence from 2005-2013 and its associations with meteorological factors in Guangdong, China
}

Cui Guo ${ }^{1}$, Lin Yang ${ }^{2}$, Chun-Quan Ou ${ }^{1 *}$, Li Li $i^{1}$, Yan Zhuang ${ }^{1}$, Jun Yang ${ }^{3}$, Ying-Xue Zhou ${ }^{1}$, Jun Qian ${ }^{4 *}$, Ping-Yan Chen ${ }^{1}$ and Qi-Yong Liu ${ }^{3}$

\begin{abstract}
Background: The temporal variation of malaria incidence has been linked to meteorological factors in many studies, but key factors observed and corresponding effect estimates were not consistent. Furthermore, the potential effect modification by individual characteristics is not well documented. This study intends to examine the delayed effects of meteorological factors and the sub-population's susceptibility in Guangdong, China.

Methods: The Granger causality Wald test and Spearman correlation analysis were employed to select climatic variables influencing malaria. The distributed lag non-linear model (DLNM) was used to estimate the non-linear and delayed effects of weekly temperature, duration of sunshine, and precipitation on the weekly number of malaria cases after controlling for other confounders. Stratified analyses were conducted to identify the subpopulation's susceptibility to meteorological effects by malaria type, gender, and age group.
\end{abstract}

Results: An incidence rate of 1.1 cases per 1,000,000 people was detected in Guangdong from 2005-2013. High temperature was associated with an observed increase in malaria incidence, with the effect lasting for four weeks and a maximum relative risk (RR) of 1.57 (95\% confidence interval (Cl): $1.06-2.33$ ) by comparing $30^{\circ} \mathrm{C}$ to the median temperature. The effect of sunshine duration peaked at lag five and the maximum RR was 1.36 (95\% Cl: 1.08-1.72) by comparing 24 hours/week to 0 hours/week. A J-shaped relationship was found between malaria incidence and precipitation with a threshold of $150 \mathrm{~mm} /$ week. Over the threshold, precipitation increased malaria incidence after four weeks with the effect lasting for 15 weeks, and the maximum RR of 1.55 (95\% Cl: 1.18-2.03) occurring at lag eight by comparing $225 \mathrm{~mm} /$ week to $0 \mathrm{~mm} /$ week. Plasmodium falciparum was more sensitive to temperature and precipitation than Plasmodium vivax. Females had a higher susceptibility to the effects of sunshine and precipitation, and children and the elderly were more sensitive to the change of temperature, sunshine duration, and precipitation.

Conclusion: Temperature, duration of sunshine and precipitation played important roles in malaria incidence with effects delayed and varied across lags. Climatic effects were distinct among sub-groups. This study provided helpful information for predicting malaria incidence and developing the future warning system.

Keywords: Distributed lag non-linear model, Malaria incidence, Temperature, Duration of sunshine, Precipitation

\footnotetext{
* Correspondence: ouchunquan@hotmail.com; qianjun_gz@126.com

${ }^{1}$ State Key Laboratory of Organ Failure Research, Department of Biostatistics, School of Public Health and Tropical Medicine, Southern Medical University, Guangzhou 510515, China

${ }^{4}$ Department of Mathematics and Physics, School of Biomedical Engineering, Southern Medical University, Guangzhou 510515, China

Full list of author information is available at the end of the article
} 


\section{Background}

Malaria, one of the major global public health threats, has high morbidity and mortality despite relevant effective control in recent decades [1]. Globally, there were an estimated 207 million cases of malaria in 2012, and an increasing trend was observed in the annual numbers of malaria deaths $[2,3]$. It is well recognized that the prevalence and transmission of malaria are associated with multiple factors, such as meteorological, demographic and socio-economic factors, human behaviours that involve migration, excessive urbanization and air travel, and biological factors such as type of malaria vector and mosquito density [4-9]. Previous data have shown the apparent seasonal cycle of one year. The malaria incidence peaks in summer and autumn in most places, including China and Africa, and sometimes relatively small peaks can be seen during January and February $[5,10,11]$. This seasonal feature is dominantly driven by meteorological conditions, which affect mosquito abundance, feeding habits and life cycle period, and the parasite's interaction with mosquitoes (e.g., the duration of sporogony) [6]. Some previous studies have shown that the intra-annual variation in malaria incidence may be associated with changes in ambient temperature, duration of sunshine, precipitation, wind speed, and relative humidity, etc., but there were inconsistent findings in the key factors observed and the corresponding effect estimates. For example, some studies reported significant effects of precipitation on malaria cases $[4,6,10]$, while others found that the effects were governed by temperature and sunshine or no effects of precipitation were observed $[7,12,13]$. This discrepancy may be partly due to regional heterogeneity in climate and environment and different assumptions of various models (e.g., linear or non-linear effects assumed).

The climatic factors mainly affect the densities and activities of mosquitoes and the development of Plasmodium in mosquitoes, and there is an incubation period of seven days or longer between the bite of an infected mosquito and the onset of malaria symptoms [14]. Therefore, the effects of meteorological factors on malaria may be delayed. Many epidemiological studies assumed a linear relationship between climatic parameters and malaria [5,11], while it was not well documented whether there is a potential non-linear relationship. Most previous studies on environmental factors and malaria have adopted an autoregressive integrated moving average (ARIMA) model and its derived methods (SARIMA, GSARIMA and ARIMAX), which elucidated how climate could impact malaria and gave clues to prediction, but their applications were restricted by specific lags [15-18]. Several studies used regression analyses such as linear regression, logistic regression and a linear mixed model, which completely ignored the lagged effects of meteorological factors $[5,19,20]$. Recently, the distributed lag non-linear model (DLNM) has been applied in several studies $[4,7,9,10]$, while uncertainties remain about potential effect modification by malaria type and individual characteristics, such as gender and age.

Malaria remains a serious health threat in China, particularly in southern China. The sub-tropical and tropical climate, accompanied by fast development of economic and population mobility, provide a favourable environment for Plasmodium and mosquitoes throughout the year, resulting in a potential threat of outbreak [4,10,11,21]. Guangdong, the largest and wealthiest province in southern China, suffered a lot from malaria in the past, but few epidemiologic studies of malaria have been conducted. This study aims to describe the epidemiologic features of malaria, investigate the non-linear lagged effects of climatic factors on malaria, and examine potential effect modification by individual characteristics in Guangdong, China.

\section{Methods}

\section{Study site}

Guangdong Province is situated at the southernmost tip of mainland China $\left(20^{\circ} 18^{\prime}\right.$ to $25^{\circ} 28^{\prime} \mathrm{N}, 107^{\circ} 25^{\prime}$ to $\left.109^{\circ} 45^{\prime} \mathrm{E}\right)$ adjacent to Hong Kong (Figure 1). Guangdong has a population of 106 million residing in a land area of 179,612 sq $\mathrm{km}$ and a sub-tropical, marine, monsoon climate with an annual average temperature of $22^{\circ} \mathrm{C}$ and annual average rainfall of $1,500 \mathrm{~mm}$.

\section{Data collection}

In mainland China, each individual case of notable disease including malaria, must be reported to the Chinese Centre for Disease Control and Prevention (CCDC) through the online Infection Diseases Monitor Information System. The obtained data of all reported malaria cases in Guangdong during the period from 1 January, 2005 to 31 December, 2013 was obtained. The weekly number of cases was aggregated in this study. Demographic data of Guangdong used in this study were collected from the Sixth China Population Census in 2010.

The China Meteorological Data Sharing Service System provided daily data of meteorological measures collected at 36 weather stations in Guangdong from 2005 to 2013. Weekly average/minimum/maximum temperature $\left({ }^{\circ} \mathrm{C}\right)$, cumulative duration of sunshine (hours/week), cumulative precipitation ( $\mathrm{mm} /$ week), average/maximum/extreme wind speed $(\mathrm{m} / \mathrm{s})$, average/minimum/maximum atmospheric pressure $(\mathrm{hPa})$, and average relative humidity (\%) were calculated from all of the stations as meteorological measures for the whole territory of Guangdong.

\section{Statistical analyses}

The Granger causality Wald test was performed to examine whether climatic factors could predict malaria incidence (Additional file 1). This method was applied to 


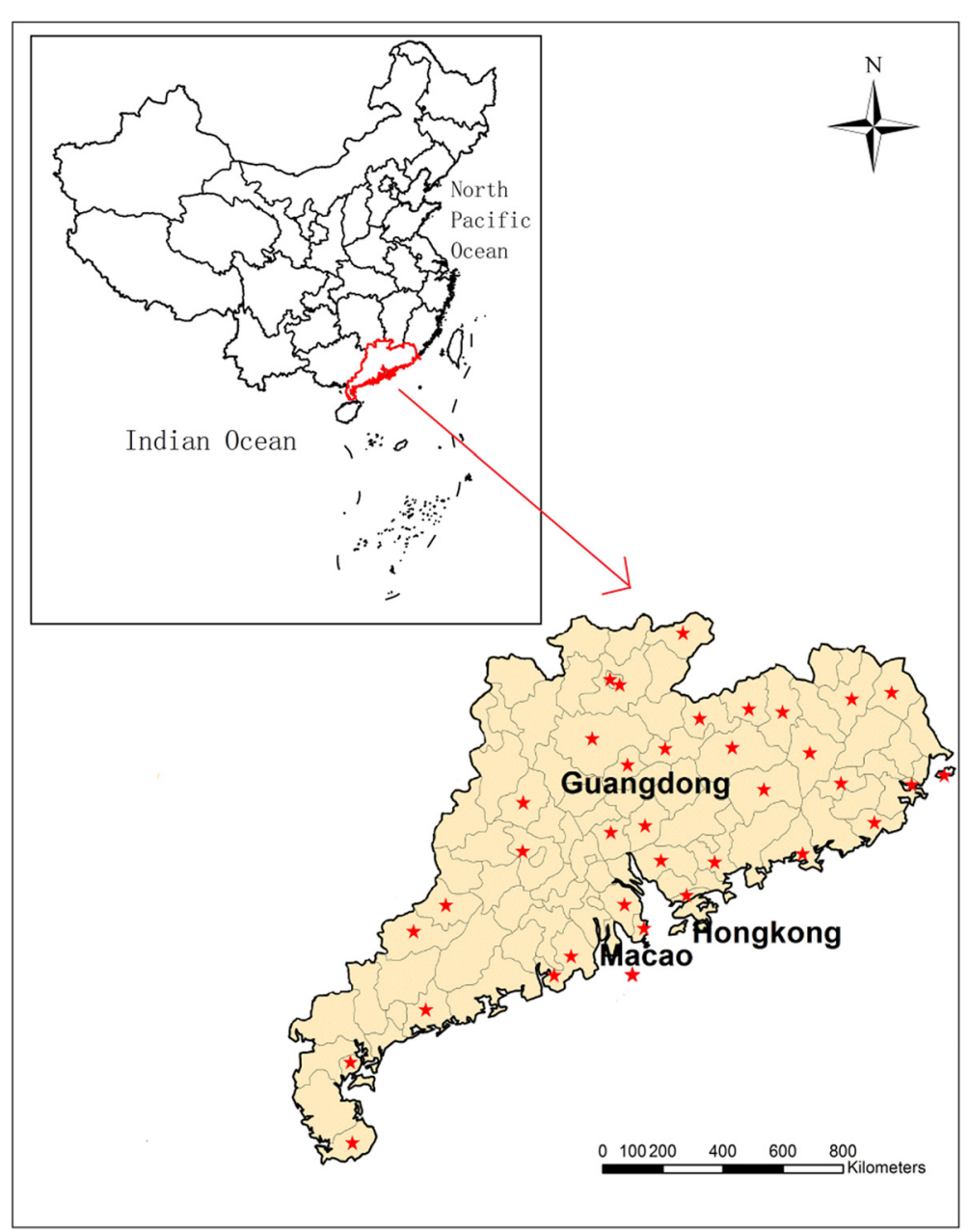

Figure 1 Map of Guangdong. The stars show the locations of weather stations in Guangdong.

investigate whether one time-series could predict another, and this association would be invalid when the two series were reversed [22,23]. Statistically significant factors were selected for the following correlation analyses. Spearman correlation analyses were performed to examine the pair-wise correlation among different meteorological factors. To avoid multicollinearity, the highly correlated meteorological factors $\left(r_{s}>0.7\right)$ were not included in the regression model simultaneously [7]. In the final model, weekly average temperature, duration of sunshine and precipitation were considered simultaneously. They may provide different mechanism for malaria transmission and be controlled for while assessing the effects of the other factor.

DLNM was applied to estimate the effects of weekly climatic factors on the weekly number of malaria cases. DLNM is a modelling framework that flexibly describes the non-linear and lagged effects of exposures, and it was originally used in time series analysis [22]. This modelling method has been used widely to assess climatic effects on mortality and morbidity [22,24]. DLNM could simultaneously examine the climate effects over lag and meteorological factors. Gasparrini [25] provided a thorough methodological introduction of DLNM. This study aimed to detect the short-term effects of meteorological factors on malaria cases. Therefore, it is important to control for seasonality and the long-term trend of malaria. Because the weekly number of malaria cases shows an apparent 1-year period (i.e., 52 weeks), the Fourier transformation was used to control for seasonality, as used by Kim et al. [7]. The analysis showed that this method produced minimum Q-AIC and had fewer parameters to be estimated compared with the spline method used in a previous study [26]. The long-term trend was controlled for by the inclusion of a linear term of year (year $=2005,2006 \ldots$ 2013) $[7,26]$. To check whether the malaria incidence has a nonlinear trend across years, a quadratic term of year was added into the model and found that the goodness-of-fit 
of the model was not improved significantly, suggesting that a linear function of year is enough to demonstrate the long-term trend.

The model can be specified as follows:

$$
\begin{aligned}
\log \left(E\left(Y_{t}\right)\right)=\alpha & +\sum_{l=0}^{10} f\left(T_{(t-l)} ; \alpha_{l}\right) \\
& +\sum_{l=0}^{10} f\left(S_{(t-l)} ; \gamma_{l}\right)+\sum_{l=0}^{15} f\left(P_{(t-l)} ; \delta_{l}\right) \\
& + \text { ns }(\text { meanwind }, 3)+\sin \left(\frac{2 \pi t}{52}\right) \\
& +\cos \left(\frac{2 \pi t}{52}\right)+\text { year }
\end{aligned}
$$

where $\alpha$ is the intercept. $Y_{t}$ is the number of malaria cases in the week $\mathrm{t}(t=1,2, \ldots, 471)$, assumed to follow the quasi-Poisson distribution. $T_{t-l}, S_{t-l}$ and $P_{t-l}$ indicate weekly average temperature, duration of sunshine and precipitation with their corresponding coefficients of $\alpha_{l}$, $\gamma_{l}$ and $\delta_{l}$, respectively. Function $f$ represents the twodimensional space of cross-basis functions of natural cubic splines with possible lags. The ranges of lag $0-10$, $0-10$ and $0-15$ weeks were taken to adequately detect the effects of temperature, sunshine and rainfall, respectively $[4,6,7]$. Natural cubic splines function with degrees of freedom $(d f) 3-5$ was used for climatic variables and their lags to determine the overall climate effects (Additional file 2). In the final model, the $d f$ was specified to be 3 according to the minimum value of Akaike's Information Criterion for quasi-Poisson (Q-AIC) and the simplicity of the model $[27,28]$. Relative risk (RR) of malaria morbidity was calculated with reference to the median value for temperature and zero for duration of sunshine and precipitation [4]. Sensitivity analyses were performed by varying $d f(3-5)$ for lags.

Using the same model, stratified analyses were conducted to identify subpopulation's susceptibility to meteorological effects by malaria type, gender, and age group. All data analyses were performed in R 3.0.2.

\section{Results}

During the study period of 2005-2013, 1,075 malaria cases were reported in Guangdong with an annual incidence rate of 1.1 cases per $1,000,000$ people (Table 1). Most cases were caused by Plasmodium vivax $(44.9 \%)$ and Plasmodium falciparum (30.4\%) with annual incidence rates of 0.5 and 0.3 per $1,000,000$, respectively. Some 856 cases $(79.6 \%)$ were confirmed by a laboratory test for malaria, and 219 cases (20.4\%) were clinically diagnosed (Table 1). The annual incidence rate of men was approximately 3.6 times as high as that of women, and
Table 1 Demographic characteristic of malaria cases in Guangdong, 2005-2013

\begin{tabular}{lll}
\hline Variables & Malaria cases (\%) & $\begin{array}{l}\text { Malaria annual incidence } \\
\text { (per 1 million persons) }\end{array}$ \\
\hline Type of diagnosis & & - \\
Clinical diagnosis* & $219(20.4)$ & - \\
Laboratory diagnosis $^{\#}$ & $856(79.6)$ & \\
Type of malaria & & 0.3 \\
P. falciparum malaria & $327(30.4)$ & 0.5 \\
P. vivax malaria & $483(44.9)$ & 0.3 \\
Others & $265(24.7)$ & \\
Gender & & 1.8 \\
Male & $862(80.2)$ & 0.5 \\
Female & $213(19.8)$ & \\
Age group & & 0.2 \\
Young (0-14 years) & $35(3.3)$ & 1.3 \\
Middle (15-59 years) & $964(89.7)$ & 1.2 \\
Old (> = 60 years) & $76(7.1)$ & 1.1 \\
Total & $1075(100.0)$ & \\
\hline
\end{tabular}

*Diagnosed according to clinical characteristics of patients, including fever epidemics, periodic chills, coma and convulsions, etc.

\#Diagnosed by checking Plasmodium on the blood smear of patients.

adults suffered from a particularly high risk of malaria compared with the young (0-14 years old).

The obvious one-year seasonal cycle was observed for malaria cases and all meteorological measures (Figure 2). There was a peak in malaria cases in summer and a trough in winter, corresponding to the peaks and troughs in temperature. The duration of sunshine increased dramatically from July to October, while precipitation was higher from April to September.

The Spearman correlation coefficients between the weekly number of malaria cases and mean temperature, duration of sunshine, and precipitation were $0.35(\mathrm{P}<0.001), 0.14$ $(\mathrm{P}<0.001)$ and $0.19(\mathrm{P}=0.003)$, respectively.

The three-dimensional exposure-response surfaces reveal the non-linear relationships between these three climatic factors and malaria risks (Figure 3). There were inconsistent patterns across lags. The effects of high temperatures (above the median of $23.5^{\circ} \mathrm{C}$ ) on malaria were positive and significant within the first two weeks (lag 0-2) and decreased gradually with a duration of approximately four weeks (Figures $3 \mathrm{~A}$ and $4 \mathrm{C}$ and D). There was a maximum relative risk (RR) of 1.57 (95\% confidence interval (CI): $1.06-2.33$ ) by comparing $30^{\circ} \mathrm{C}$ to the median temperature at lag 0 . The cumulative RR of malaria over lags zero to four were 1.87 (95\% confidence interval (CI): 1.12-3.11) and 2.58 (95\% CI: 1.10-6.05) by comparing the 75th and 95th to the 50th percentiles of weekly temperature, respectively (Table 2 ). The effects of low temperature (below $23.5^{\circ} \mathrm{C}$ ) were relatively small but 


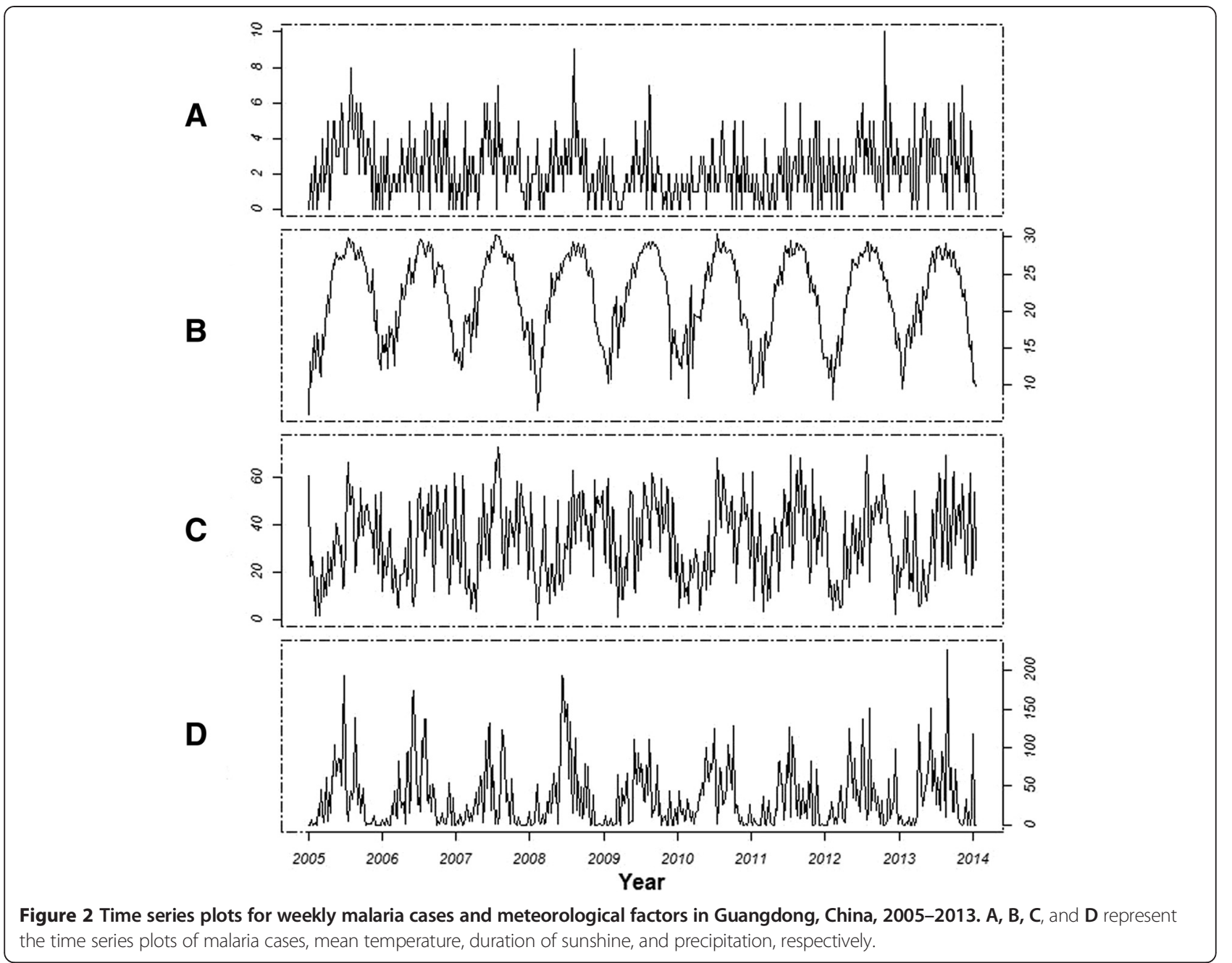

lasted for a period of ten weeks (Figures $3 \mathrm{~A}$ and $4 \mathrm{~A}$ and $\mathrm{B})$. The fifth and 25th percentiles of temperature were associated with an increase in malaria risk over lags zero to ten, when compared with the median temperature (Table 2).

A remarkable increase was observed in malaria risk associated with increased weekly duration of sunshine across the range of zero to 20 hours/week, and the risk remained stable when duration of sunshine was above 20 hours/week (Figure 3B). The effects of sunshine were delayed by two weeks, and peaked at lag 5 with a RR of $1.4(95 \%$ CI: $1.1-1.7)$ when the sunshine duration was 24 hours/ week. Figure $4(\mathrm{E}-\mathrm{H})$ shows an inverse U-shaped curve

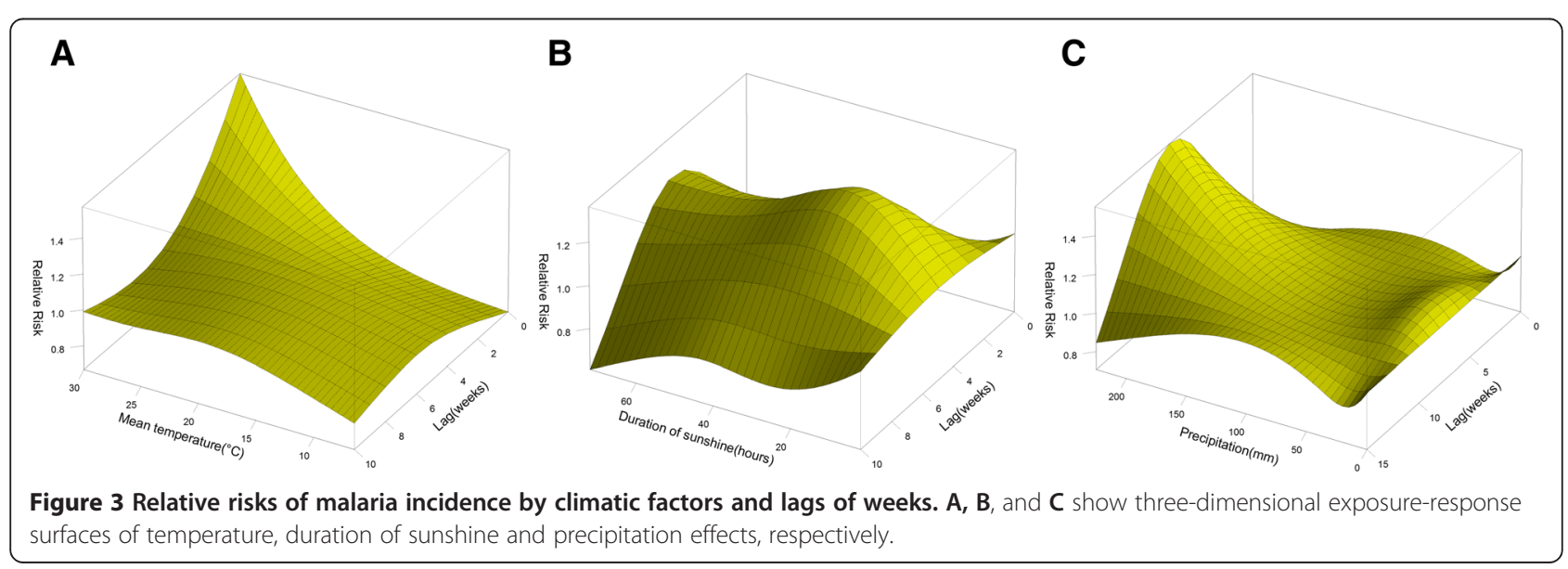




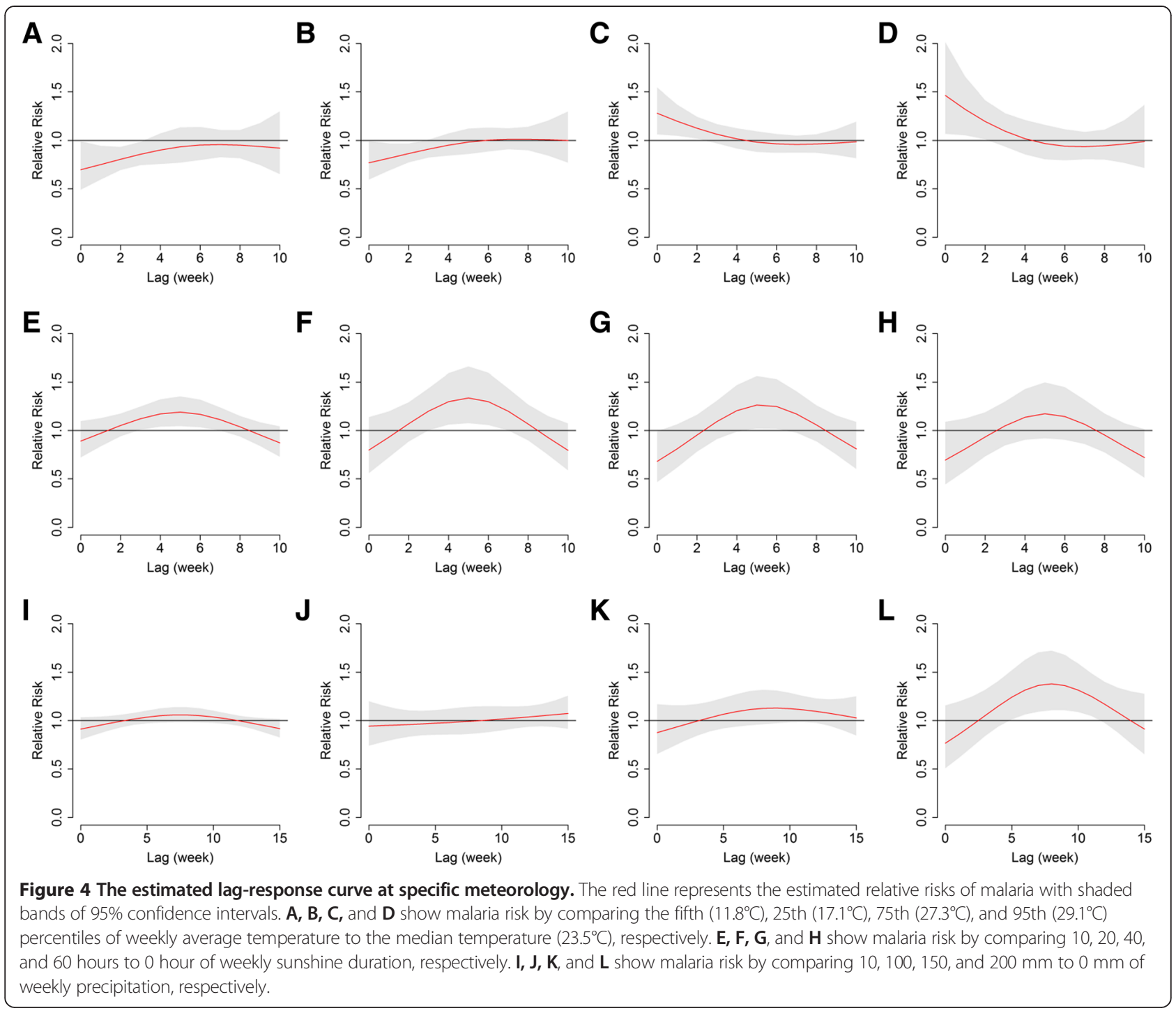

of sunshine-associated malaria risk across lags. When weekly sunshine duration was shorter than 40 hours/ week, an increase in sunshine hours was associated with a significant increase in malaria incidence in the following three to seven weeks, while the effects were not statistically significant when the duration was over 40 hours/week.

The malaria risk increased with the ascent of weekly precipitation when the precipitation was larger than $100 \mathrm{~mm} /$ week. The effects were delayed by four weeks and peaked at lag 8 with a RR of 1.5 (95\% CI: 1.2-2.0) when the precipitation was $225 \mathrm{~mm} /$ week (Figure 3C). Figure $4 \mathrm{~K}$ and $\mathrm{L}$ show similar non-linear trends of rainfall-related risk across lags for different precipitation over $150 \mathrm{~mm} /$ week. When weekly precipitation was beyond $150 \mathrm{~mm} /$ week, the rise was associated with a significant increase in malaria incidence at the lag of five to 12 weeks, while the effects were not statistically significant below $150 \mathrm{~mm} /$ week.
The stratified analyses were conducted by malaria type, gender, and age group. The three-dimensional exposureresponse surfaces (Figures 5, 6 and 7) illustrate discrepancies in meteorological effects among different malaria sub-types and sub-groups. The number of $P$. falciparum malaria was affected more by the variations in temperature and precipitation compared with $P$. vivax malaria (Figures 5 and 7). Interestingly, sunshine had negative effects on $P$. falciparum malaria and the RR decreased with the increase of sunshine duration (Figure 6). Males were at a similar temperature-related risk of malaria as females (Figure 5), while females were at a higher risk of sunshine- and precipitation-related malaria than males and the RR peaked without lag when the duration of sunshine was longer than 60 hours/week (Figures 6 and 7). The vulnerability of children and the elderly to the effects of temperature, duration of sunshine, and rainfall was consistently observed (Figures 5, 6 and 7). 
Table 2 The effects of meteorological factors on malaria cases along the lag weeks

\begin{tabular}{|c|c|c|c|c|c|c|}
\hline & \multicolumn{6}{|c|}{ Relative risk (95\% confidence interval) } \\
\hline & $\operatorname{Lag} 0$ & Lag 4 & Lag 8 & Lag $0-4^{\#}$ & Lag $0-8^{\#}$ & $\operatorname{Lag} 0-10 / 0-15^{\#}$ \\
\hline \multicolumn{7}{|c|}{ Mean temperature $\left({ }^{\circ} \mathrm{C}\right)$ (reference value: median) } \\
\hline 11.8 & $0.70(0.49,0.99)^{*}$ & $0.90(0.76,1.07)$ & $0.95(0.82,1.11)$ & $0.32(0.15,0.70)^{*}$ & $0.26(0.10,0.72)^{*}$ & $0.23(0.06,0.80)^{*}$ \\
\hline 17.1 & $0.77(0.59,0.99)^{*}$ & $0.95(0.84,1.07)$ & $1.01(0.90,1.14)$ & $0.47(0.26,0.85)^{*}$ & $0.47(0.21,1.05)$ & $0.48(0.17,1.36)$ \\
\hline 27.3 & $1.28(1.06,1.55)^{*}$ & $1.02(0.92,1.13)$ & $0.96(0.87,1.06)$ & $1.87(1.12,3.11)^{*}$ & 1.63(0.82,3.24) & $1.57(0.70,3.50)$ \\
\hline 29.1 & $1.47(1.07,2.01)^{*}$ & $1.02(0.86,1.21)$ & $0.94(0.80,1.11)$ & $2.58(1.10,6.05)^{*}$ & $2.07(0.67,6.39)$ & $1.98(0.54,7.23)$ \\
\hline \multicolumn{7}{|c|}{ Duration of sunshine (hours/week) (reference value: 0) } \\
\hline 10 & $0.89(0.72,1.10)$ & $1.17(1.04,1.32)^{*}$ & $1.04(0.94,1.15)$ & $1.20(0.68,2.10)$ & $1.92(0.88,4.21)$ & $1.60(0.66,3.85)$ \\
\hline 20 & $0.80(0.56,1.14)$ & $1.30(1.06,1.59)^{*}$ & $1.07(0.90,1.27)$ & $1.24(0.47,3.23)$ & $2.75(0.73,10.38)$ & $2.02(0.45,9.04)$ \\
\hline 40 & $0.68(0.47,0.99)^{*}$ & $1.21(0.99,1.47)$ & $1.06(0.89,1.26)$ & $0.69(0.25,1.92)$ & $1.35(0.34,5.42)$ & $1.02(0.21,5.01)$ \\
\hline 60 & $0.69(0.44,1.09)$ & $1.14(0.90,1.43)$ & $0.95(0.77,1.17)$ & $0.63(0.18,2.16)$ & $0.85(0.17,4.40)$ & $0.51(0.08,3.29)$ \\
\hline \multicolumn{7}{|c|}{ Precipitation (mm/week) (reference value: 0 ) } \\
\hline 10 & $0.91(0.80,1.03)$ & $1.02(0.96,1.08)$ & $1.06(0.98,1.14)$ & $0.83(0.56,1.25)$ & $1.02(0.60,1.72)$ & $0.94(0.49,1.81)$ \\
\hline 100 & $0.94(0.74,1.20)$ & $0.97(0.85,1.10)$ & $1.00(0.87,1.15)$ & $0.79(0.34,1.82)$ & $0.74(0.23,2.35)$ & $0.97(0.21,4.39)$ \\
\hline 150 & $0.88(0.66,1.17)$ & $1.03(0.89,1.20)$ & $1.13(0.96,1.32)$ & $0.78(0.30,2.09)$ & $1.15(0.31,4.26)$ & $2.06(0.39,10.81)$ \\
\hline 200 & $0.77(0.51,1.16)$ & $1.15(0.94,1.41)$ & $1.38(1.11,1.72)^{*}$ & $0.76(0.19,2.96)$ & $2.33(0.39,13.95)$ & $6.00(0.61,58.96)$ \\
\hline
\end{tabular}

"The cumulative RRs within lag period.

*Relative risks are statistically significant. $11.8,17.1,27.3$, and $29.1^{\circ} \mathrm{C}$ correspond to the 5 th, 25 th, 75 th, and 95 th percentile of weekly average temperature, respectively.

Sensitivity analyses on the $d f$ for lag indicated that the final model was appropriate based on the parsimony with smaller Likelihood Akaike information criteria for quasi-Poison (Q-AIC) value and the stability (Additional file 3).

\section{Discussion}

A much higher malaria incidence rate was found in males than in females in Guangdong, and it was confirmed in the literature that children had lower malaria incidence than adults $[2,29]$. The changing epidemiology of malaria

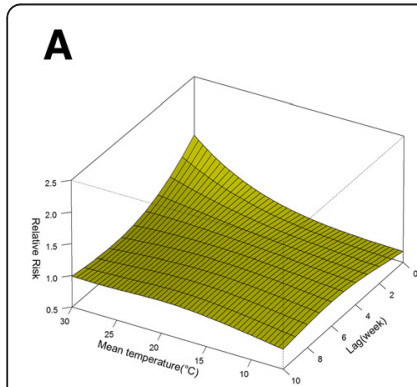

E
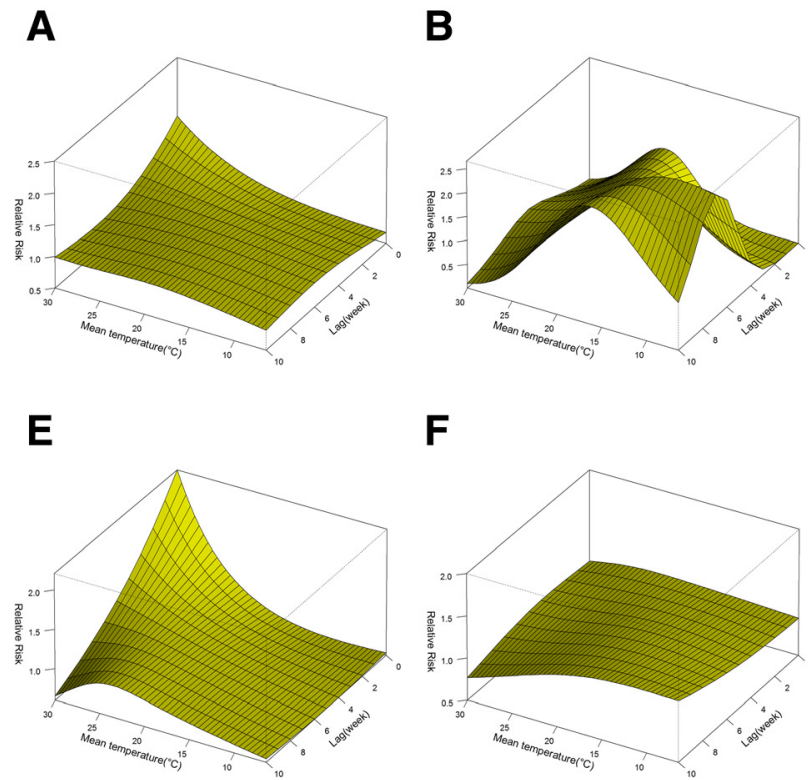

$\mathbf{F}$

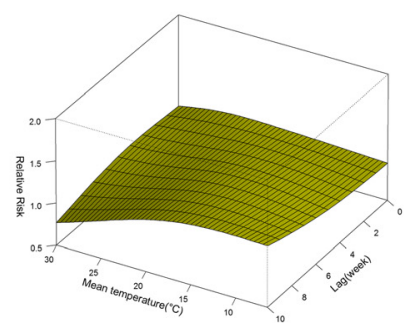

C

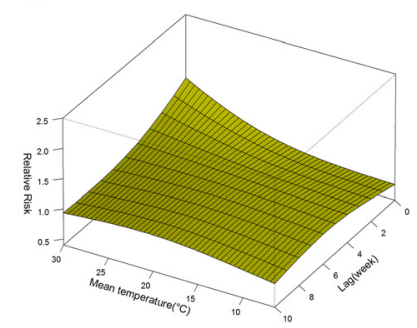

G

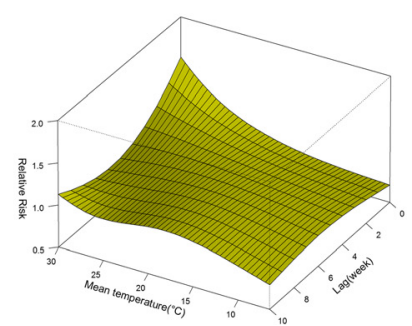

D

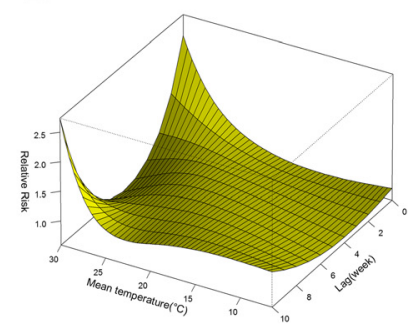

H

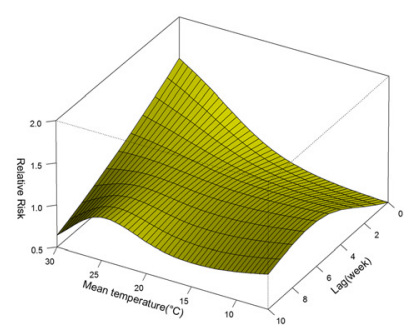

Figure $\mathbf{5}$ The relative risks of malaria morbidity by weekly average temperature and lags for subgroups. A, B, C, D, E, F, G, and $\mathbf{H}$ show three-dimensional exposure-response surfaces of temperature for the whole population, young, middle-aged, the elderly, Plasmodium falciparum malaria, Plasmodium vivax malaria, males, and females, respectively. The reference vale was median temperature $\left(23.5^{\circ} \mathrm{C}\right)$. 


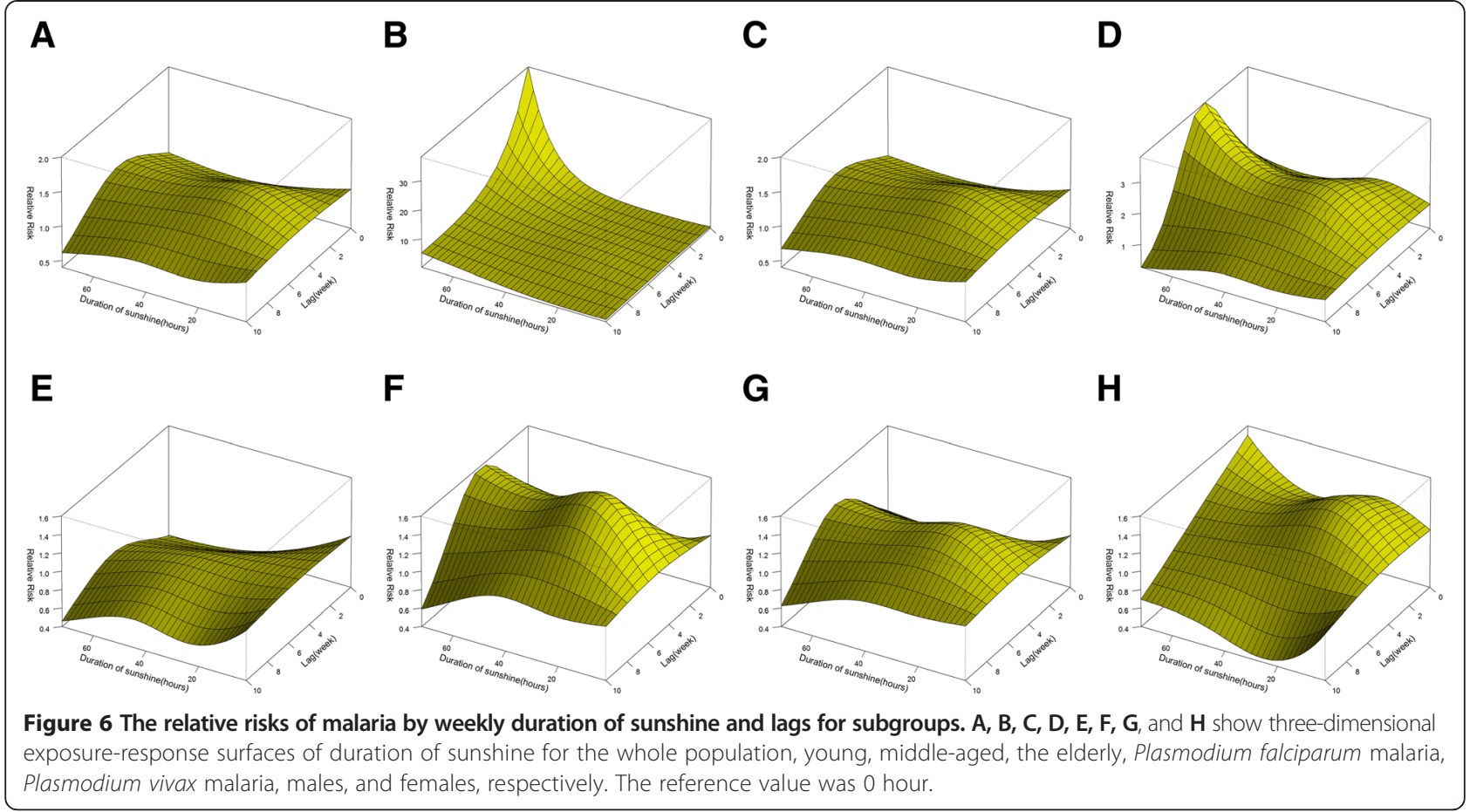

infection with an increasing proportion of adults and male cases was seen in 34 malaria-eliminating countries [30]. This indicates the increasing importance of outdoor occupational and behavioural factors that put adults, particularly male adults, at much higher chance of endemic exposure to malaria infection compared with children and women. Elderly individuals had similar incidence as the middle-aged people, probably because they had less opportunity for endemic exposure but poorer physiological functions. Plasmodium vivax malaria was likely to relapse, which may have a potential influence on the estimate of climatic effects. However, the World Health Organization

\section{A}

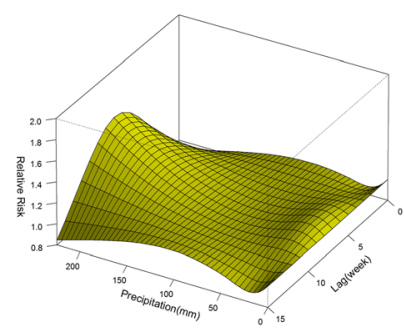

E

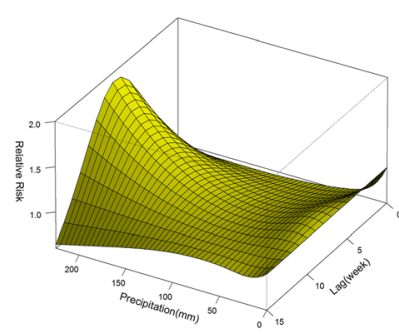

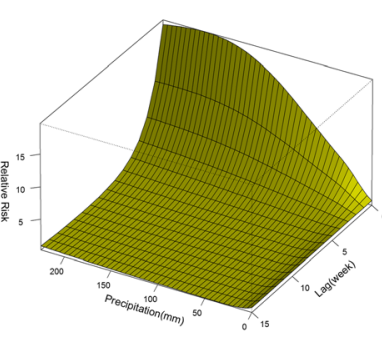

$\mathbf{F}$

B

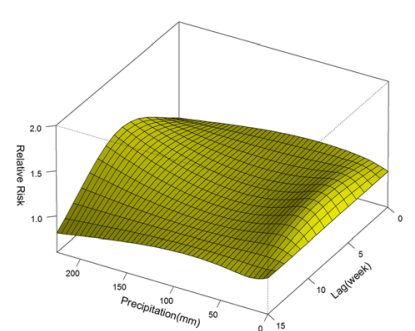

C

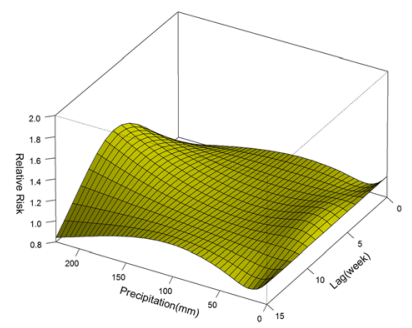

G

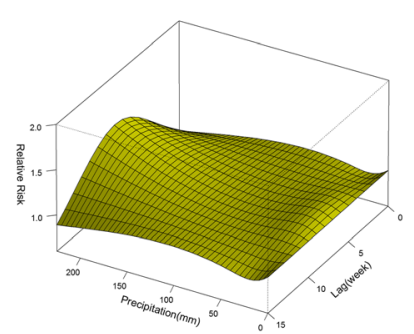

D

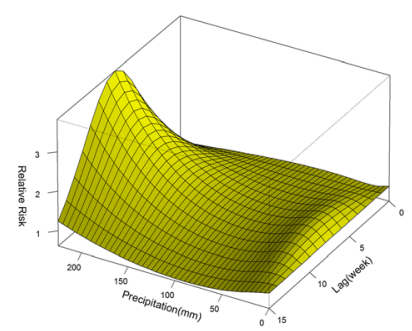

H

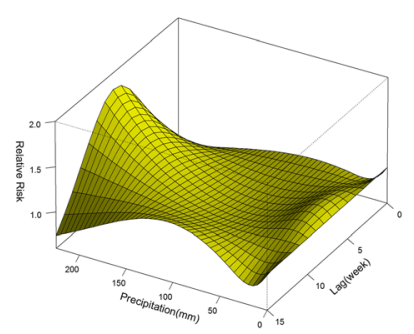

Figure 7 The relative risks of malaria by weekly precipitation and lags for subgroups. A, B, C, D, E, F, G, and $\mathbf{H}$ show three-dimensional exposure-response surfaces of precipitation for the whole population, young, middle-aged, the elderly, Plasmodium falciparum malaria, Plasmodium vivax malaria, males, and females, respectively. The reference value was $0 \mathrm{~mm}$. 
[31] noted that the relapse rate of malaria would be reduced to $0.6 \%$ if patients were treated well. In the present study, the authors believed the small relapse rate of malaria in the data (approximately 1.9\%) may not substantially bias the results for the estimation of climatic effects.

The Granger causality Wald test revealed that the mean, minimum, and maximum temperature were all predictors of malaria incidence. The mean temperature could provide more easily interpreted results in a policy context and is more familiar to the public. It is widely used as an indicator of temperature in studies of relationships between vector-borne diseases and climate [7,10,22]. However, some studies used minimum temperature, maximum temperature, or both as main predictors, which may promote examining the effects of extreme temperature, but there were strong correlations between these temperature measures, and the simultaneous inclusion of these measures in the model may complicate the model with higher Q-AIC value and invalidate the parameter estimation $[7,10,32]$. In the present study, weekly average temperature as the only temperature parameter was included in the model to assess the temperature effect on malaria incidence. The positive and non-linear relationship was found between mean temperature and malaria incidence. The immediate effects of high temperature (above $23.5^{\circ} \mathrm{C}$ ) had a duration of four weeks, while low temperature (below $23.5^{\circ} \mathrm{C}$ ) was associated with an increase in malaria risk within the following zero to ten weeks. The biological mechanism for the different time responses to high and low temperature remains unclear. Similarly, many studies have showed that the effects of low temperature on human mortality persisted for much longer than that of high temperature [33,34]. It is possible that high temperature (over the median temperature) mainly affects mosquito feeding habits and activity behaviours and the development of parasites in mosquitoes within a short time, while low temperature inhibits the survival of mosquitoes and fundamentally reduces the possibility of malaria infection for a longer period. Rueda et al. [35] noted that high temperature could decrease the body size, increase head capsule width, lengthen the larval body, add weight to the instars or pupae of mosquitoes, and increase the survival from eclosion to adult. Increasing temperature within proper scope can speed up the spreading of malaria vectors, shorten the growth period of parasites and cause fast transmission and high malaria incidence [15]. However, it is necessary to be cautious to explain and generalize the lag 0 effect of temperature. It is speculated that climate factors may have indirect effects on malaria incidence during the incubation period. It is noted that high temperature could accelerate the activities of mosquitoes and increase the bites on human [30], which may increase the rate of malaria infection at the current week. Further experimental studies on the underlying biological mechanism would be helpful to determine the time-course of the effects of ambient temperature on malaria.

Therefore, the effects of high temperature were immediate, but effects of low temperature were delayed. The evidence suggests that some measures are needed to mitigate the effects in warm seasons, such as installing air conditioners, reducing outdoor activities, and conducting some anti-mosquito operations.

The literature showed discrepancies in the lag period of temperature effect. Several previous studies showed there was little effect of temperature after ten weeks [4,6,7], while Craig and Briët et al. [5,12] reported a lag of nine to 16 weeks, which may be caused by the specific single lags chosen and the broader time scale (e.g., monthly) used. Furthermore, the regional differences in the proportions of malaria sub-types may also contribute to these discrepancies. Additionally, some previous findings were based on simple models, including only one or two climatic factors. In the present study, the effects were evaluated after adequately controlling for seasonality, trend, and potential confounding of other meteorological factors. Further multiple-region analyses using unified modeling strategies are required to better understand the lagged effects of temperature to identify the optimum time scale of supervision and effective control.

No temperature threshold was observed for the effect on malaria in Guangdong. Similarly, Wardrop and Bi et al. $[10,32]$ did not find a temperature threshold for $P$. vivax malaria in two Chinese subtropical cities. However, Kim et al. [7] observed a temperature threshold of $-4^{\circ} \mathrm{C}$ that was an optimum temperature, above or below which the risk of malaria increased, whereas the malaria risks changed slightly above $24^{\circ} \mathrm{C}$ in Korea. This is possibly because the relatively shorter range of temperatures in sub-tropical regions and relatively smaller temperature gradient could not provide the full picture of the exposureresponse relationship. In Guangdong, the range of weekly average temperature during the study period was 6.0 to $30.5^{\circ} \mathrm{C}$ compared with -8.5 to $27.5^{\circ} \mathrm{C}$ in the Korea study [7]. Additionally, the effects of hot temperature on Plasmodium falciparum malaria were greater than on $P$. vivax malaria. P. falciparum goes through the whole life cycle within a shorter time at $28-30^{\circ} \mathrm{C}$ [36]. Hot temperature favours $P$. falciparum development and may have a significant influence on mosquito infection rates and parasite densities [37]. Plasmodium vivax could complete growth and development within a broader range of 15 to $30^{\circ} \mathrm{C}$ and therefore may be less sensitive to temperature changes than $P$. falciparum.

Previously, only Kim et al. [7] considered the delayed effects of sunshine. In the present study, a statistically significant negative effect of sunshine duration on malaria 
was found at the same week, while the effects were positive at lag three to seven weeks. Furthermore, falciparum malaria and vivax malaria showed differences in the dose-response relationship with sunshine. $\mathrm{Li}$ [38] also found that there were positive associations between the monthly duration of sunshine and malaria cases in the current month. Zhang et al. and Ayala et al. [39,40] did not find a significant relationship between sunshine and concurrent malaria cases. On the contrary, a negative monotonic impact of the duration of sunshine on malaria incidence was observed in Kim's study in Korea, where only $P$. vivax malaria was considered [7]. This discrepancy may have been caused by the differences in the types of main vectors for malaria between China and Korea. The predominant vectors for $P$. vivax are Anopheles lesteri and Anopheles sinensis in Korea [41]. The former has higher sensitivity to malaria and proved to be heliophobic [42]. However, the main vector for $P$. vivax is An. sinensis in mainland China [43]. It is evident that the development of $A n$. sinensis is positively associated with the duration of sunshine [44]. While the main vectors for P. falciparum are Anopheles minimus and Anopheles dirus, which are usually active in the shadows $[45,46]$. There is a need to further explore the effects of sunshine on specific vectors and malaria subtypes.

Precipitation was the most controversial factor in previous studies of climatic effects on malaria incidence. A study found significant rainfall effects when precipitation was 2.4 times higher than the normal level [47]. Rainfall plays an important role in the survival of mosquitoes, but it may contribute little to the saturation if there has been sufficient rainfall or the sunshine effects are considered, because a small amount of water could evaporate quickly in strong sunlight [4,7]. Wardrop et al. [10] elucidated there was no significant effect when rainfall was less than $100 \mathrm{~mm} /$ month in Yunnan, China. A study in Ethiopia found that rainfall had an important influence on malaria incidence in hot districts with an altitude lower than $1,650 \mathrm{~m}$, but not in cold districts with an altitude higher than $1,650 \mathrm{~m}$ [6]. In the present study, there were significant effects of weekly rainfall over $150 \mathrm{~mm} /$ week, approximately 4.4 times as much as the mean precipitation of $34.2 \mathrm{~mm} /$ week. This is possibly because most areas in Guangdong have advanced drainage systems so that low levels of precipitation do not facilitate the development of mosquito larval habitats or mosquito transmission and therefore have no significant effect on malaria incidence. The positive effects of precipitation observed in this study had a lag of four weeks and peaked at lag of eight weeks, similar to previous findings $[6,7]$. The lagged effects were reasonable because it takes time to gather rainfall into little pools and for mosquito to lay eggs on them, consequently affecting mosquito density and malaria incidence. Consistent with Yan et al. [32], the present study indicated that precipitation-related effects for P. falciparum and $P$. vivax were similar, but the effects were relatively higher for $P$. falciparum than the other. This is probably due to the biological difference in the parasite adaptation, mosquito habits, and the climatic sensitivity between these two types of Plasmodium.

To our knowledge, no study has investigated the potential effect of gender and age on meteorology-associated risks of malaria. In the present study, no gender difference in the sensitivity to temperature-associated malaria risk was found, but females were at a higher risk of sunshineand precipitation-related malaria. Further, it was found that the young and the elderly were more susceptible to temperature-, sunshine- and precipitation-associated malaria. Their vulnerable immune system, and higher likelihood of experiencing mosquito bites, may contribute to children's susceptibility. Loss of physical function and poorer general health may put the elderly at high risk of weather-related malaria. This is supported by several studies that noted the special vulnerability of children and old people to climate change on public health $[48,49]$.

There are several limitations of the present study. Firstly, although there is a long-standing comprehensive surveillance system of malaria in Guangdong and each case must be reported to the national $\mathrm{CCDC}$, the under-reporting of malaria cases is inevitable. Secondly, the effects of extreme weather events, such as heat waves and floods, were not considered, which may play an important role in malaria pandemics. Although the nine-year data were utilized as much as possible, the analyses on a weekly basis were performed and significant effects of some metrological factors may not have been detected because of the limited number of cases, particularly for some specific subgroups using stratified analyses. Further analyses based on a longer study period or daily data if appropriate may promote a better understanding of climatic effects.

\section{Conclusions}

Malaria remains an important public health problem in Guangdong, China. Malaria cases cluster in male adults. It was found that mean temperature, followed by weekly duration of sunshine and weekly precipitation, had delayed and non-linear effects on malaria incidence, particularly for susceptible sub-populations. The findings promote a better understanding of climatic effects on malaria and provide important information for malaria prediction. Further combinations with data from past decades or other regions and the consideration of extreme weather events could be used to construct a malaria warning system. 


\section{Additional files}

\section{Additional file 1: The results of the Granger causality Wald tests for} climate factors.

Additional file 2: Likelihood Akaike's information criteria for quasiPoison (Q-AIC) values for different models. All the dfs were selected from 3-5 for mean temperature, precipitation, duration of sunshine and mean wind with smallest Q-AIC value. The bold value refers to the Q-AIC value of the final model.

Additional file 3: Sensitive analyses with Likelihood Akaike's information criteria for quasi-Poison (Q-AIC) values for different models. The Q-AIC values of models with different degrees of freedom for lags. The bold value refers to the Q-AIC value of the final model.

\section{Competing interests}

The authors declare that they have no competing interests.

\section{Authors' contributions}

CQ and JQ initiated the study. CG, LL and YX cleaned and analysed the data. $C G, C Q$ and LY drafted the manuscript. CQ, PY, YZ, JY, QY, and JQ contributed to research design and data collection. All authors reviewed and approved the final manuscript.

\section{Acknowledgements}

This research was supported by Science and Technology Planning Project of Guangdong Province, China (2013B021800041) and the National Natural Science Foundation of China (81102207).

\section{Author details}

${ }^{1}$ State Key Laboratory of Organ Failure Research, Department of Biostatistics, School of Public Health and Tropical Medicine, Southern Medical University, Guangzhou 510515, China. ${ }^{2}$ Department of Nursing, Faculty of Health and Social Sciences, The Hong Kong Polytechnic University, Hong Kong, China ${ }^{3}$ State Key Laboratory for Infectious Disease Prevention and Control, National Institute for Communicable Disease Control and Prevention, Chinese Center for Disease Control and Prevention, Beijing 102206, China. ${ }^{4}$ Department of Mathematics and Physics, School of Biomedical Engineering, Southern Medical University, Guangzhou 510515, China.

Received: 27 November 2014 Accepted: 1 March 2015

Published online: 18 March 2015

\section{References}

1. Vitoria M, Granich R, Gilks CF, Gunneberg C, Hosseini M, Were W, et al. The global fight against HIV/AIDS, tuberculosis, and malaria. Am J Clin Pathol. 2009:131:844-8.

2. Murray CJL, Rosenfeld LC, Lim SS, Andrews KG, Foreman KJ, Haring D, et al. Global malaria mortality between 1980 and 2010: a systematic analysis. Lancet. 2012;379:413-31.

3. WHO. World Malaria Report 2013. Geneva, Switzerland: World Health Organization; 2013

4. Zhao X, Chen F, Feng Z, Li XS, Zhou XH. The temporal lagged association between meteorological factors and malaria in 30 counties in south-west China: a multilevel distributed lag non-linear analysis. Malar J. 2014;13:57.

5. Craig MH, Kleinschmidt I, Nawn JB, Sueur DL, Sharp BL. Exploring 30 years of malaria case data in KwaZulu-Natal, South Africa: Part I. The impact of climatic factors. Trop Med Int Health. 2004;9:1247-57.

6. Teklehaimanot HD, Lipsitch M, Teklehaimanot A, Schwartz J. Weather-based prediction of Plasmodium falciparum malaria in epidemic-prone regions of Ethiopia I. Patterns of lagged weather effects reflect biological mechanisms. Malar J. 2004;3:41.

7. Kim YM, Park JW, Cheong HK. Estimated effect of climatic variables on the transmission of Plasmodium vivax malaria in the Republic of Korea. Environ Health Persp. 2012:120:1314-9.

8. Babu MU, Puttaiah ET. Socio-economic implications of climate change: a study on vector borne diseases (malaria) at micro level. Karnataka: Gulbarga University; 2012.

9. Chatterjee C, Sarkar RR. Multi-step polynomial regression method to model and forecast malaria incidence. PLoS One. 2009:4:e4726.
10. Wardrop NA, Barnett AG, Atkinson J, Clements AC. Plasmodium vivax malaria incidence over time and its association with temperature and rainfall in four counties of Yunnan province. China Malar J. 2013;12:452.

11. Bi Y. Impact of socio-ecological variability on the transmission of malaria in Yunnan Province China. Brisbane: Queensland University of Technology; 2013.

12. Briët OJ, Vounatsou P, Gunawardena DM, Galappaththy GN, Amerasinghe $\mathrm{PH}$. Models for short term malaria prediction in Sri Lanka. Malar J. 2008;7:76.

13. Bouma MJ, Dye C, Van Der Kaay HJ. Falciparum malaria and climate change in the Northwest Frontier province of Pakistan. Am J Trop Med Hyg. 1996:55:131-7.

14. WHO. Malaria. In: International Travel and Health: Disease Information. Geneva, Switzerland: World Health Organization; 2014.

15. Loevinsohn ME. Climatic warming and increased malaria incidence in Rwanda. Lancet. 1994;343:714-8.

16. Bi P, Tong S, Donald K, Parton KA, Ni J. Climatic variables and transmission of malaria: a 12-year data analysis in Shuchen county, China. Public Health Rep. 2003;118:65-71.

17. Briët OJ, Amerasinghe PH, Vounatsou P. Generalized seasonal autoregressive integrated moving average models for count data with application to malaria time series with low case numbers. PLoS One. 2013;8:e65761.

18. Huang F, Zhou S, Zhang S, Wang H, Tang L. Temporal correlation analysis between malaria and meteorological factors in Motuo county, Tibet. Malar J. 2011:10:54.

19. Eisele TP, Larsen DA, Anglewicz PA, Keating J, Yukich J, Bennett A, et al. Malaria prevention in pregnancy, birthweight, and neonatal mortality: a meta-analysis of 32 national cross-sectional datasets in Africa. Lancet Infect Dis. 2012;12:942-9.

20. Abeku TA, De Vlas SJ, Borsboom GJJM, Tadege A, Gebreyesus Y, et al. Effects of meteorological factors on epidemic malaria in Ethiopia: a statistical modeling approach based on theoretical reasoning. Parasitology. 2004;128:585-93.

21. Wen L, Li C, Lin M, Yuan Z, Huo D, Li S, et al. Spatio-temporal analysis of malaria incidence at the village level in a malaria-endemic area in Hainan. China Malar J. 2011;10:88

22. Eisenberg $M C$, Kujbida G, Tuite AR, Fisman DN, Tien JH. Examining rainfall and cholera dynamics in Haiti using statistical and dynamic modeling approaches. Epidemics. 2013:5:197-207.

23. Kuster SP, Tuite AR, Kwong JC, McGeer A, Fisman DN. Evaluation of coseasonality of influenza and invasive pneumococcal disease: results from prospective surveillance. PLoS Med. 2011;8:e1001042.

24. Breitner S, Wolf K, Devlin RB, Diaz-Sanchez D, Peters A, Schneider A. Short-term effects of air temperature on mortality and effect modification by air pollution in three cities of Bavaria, Germany: a time-series analysis. Sci Total Environ. 2014:485-486:49-61.

25. Gasparrini A. Distributed lag linear and non-linear models in $\mathrm{R}$ : the package dlnm. J Stat Softw. 2011:43:1.

26. Bhaskaran K, Gasparrini A, Hajat S, Smeeth L, Armstrong B. Time series regression studies in environmental epidemiology. Int J Epidemiol. 2013;42:1187-95.

27. Peng RD, Dominici F, Louis TA. Model choice in time series studies of air pollution and mortality. J R Statist Soc A. 2006;169:179-203.

28. Duncombe J, Clements A, Davis J, Hu W, Weinstein P, Ritchie S. Spatiotemporal patterns of Aedes aegypti populations in Cairns, Australia: assessing drivers of dengue transmission. Trop Med Int Health. 2013;18:839-49.

29. Islam N, Bonovas S, Nikolopoulos GK. An epidemiological overview of malaria in Bangladesh. Travel Med Infect Di. 2013;11:29-36.

30. Cotter C, Sturrock HJW, Hsiang MS, Liu J, Phillips AA, Hwang J, et al. The changing epidemiology of malaria elimination: new strategies for new challenges. Lancet. 2013;382:900-11.

31. WHO. Guidelines for the treatment of malaria. Geneva: World Health Organization; 2006

32. Bi Y, Yu WW, Hu WB, Lin HL, Guo YM, Zhou XN, et al. Impact of climate variability on Plasmodium vivax and Plasmodium falciparum malaria in Yunnan Province. China Parasit Vectors. 2013:6:357.

33. Yang J, Liu HZ, Ou CQ, Lin GZ, Zhou Q, Shen GC, et al. Global climate change: Impact of diurnal temperature range on mortality in Guangzhou, China. Environ Pollut. 2013;175:131-6.

34. Guo YM. Estimation the effects of ambient temperature on mortality: methodological challenges and proposed solutions. Brisbane: Queensland University of Technology; 2012.

35. Rueda LM, Patel KJ, Axtell RC, Stinner RE. Temperature-dependent development and survival rates of Culex quinquefasciatus and Aedes aegypti (Diptera: Culicidae). J Med Entomol. 1990;27:892-8. 
36. Stratman-Thomas WK. The influence of temperature on Plasmodium vivax. Am J Trop Med Hyg. 1940;20:703-15.

37. Noden BH, Kent MD, Beier JC. The impact of variations in temperature on early Plasmodium falciparum development in Anopheles stephensi. Parasitology. 1995;111:539-45.

38. Li T, Yang Z, Wang M. Temperature, relative humidity and sunshine may be the effective predictors for occurrence of malaria in Guangzhou, southern China, 2006-2012. Parasit Vectors. 2013;6:155.

39. Zhang Y, Liu QY, Luan RS, Liu XB, Zhou GC, Jiang JY, et al. Spatial-temporal analysis of malaria and the effect of environmental factors on its incidence in Yongcheng, China, 2006-2010. BMC Public Health. 2012;12:544.

40. Ayala D, Costantini C, Ose K, Kamdem GC, Antonio-Nkondjio C, Agbor JP, et al. Habitat suitability and ecological niche profile of major malaria vectors in Cameroon. Malar J. 2009;8:307.

41. Joshi D, Choochote W, Park MH, Kim JY, Kim TS, Suwonkerd W, et al. The susceptibility of Anopheles lesteri to infection with Korean strain of Plasmodium vivax. Malar J. 2009;8:42.

42. Sinka ME, Bangs MJ, Manguin S, Chareonviriyaphap T, Patil AP, Temperley WH, et al. The dominant Anopheles vectors of human malaria in the Asia-Pacific region: occurrence data, distribution maps and bionomic précis. Parasit Vectors. 2011:4:89.

43. Makhawi AM, Liu XB, Yang SR, Liu QY. Genetic variations of ND5 gene of mtDNA in populations of Anopheles sinensis (Diptera: Culicidae) malaria vector in China. Parasit Vectors. 2013;6:290.

44. Ree HI. Studies on Anopheles sinensis, the vector species of vivax malaria in Korea. Korean J Parasitol. 2005:43:75-92.

45. Trung HD, Bortel WW, Sochantha T, Keokenchanh K, Quang NT, Cong LD, et al. Malaria transmission and major malaria vectors in different geographical areas of Southeast Asia. Trop Med Int Health. 2004;9:230-7.

46. Oo TT. The Biology and Vector Competence of the Anopheline Mosquitoes of Myanmar With Special Consideration of Anopheles Dirus. Heidelberg: Ruperto-Carola University of Heidelberg, Germany; 2003.

47. Lindsay SW, Bødker R, Malima R, Msangeni HA, Kisinza W. Effect of 1997-98 El Niño on highland malaria in Tanzania. Lancet. 2000;355:989-90.

48. Bush KF, Luber G, Kotha SR, Dhaliwal RS, Kapil V, Pascual M, et al. Impacts of climate change on public health in India: future research directions. Environ Health Persp. 2011;119:765-70.

49. Fund UNC. Children's Vulnerability to Climate Change and Disaster Impacts in East Asia and the Pacific. Bangkok: UNICEF East Asia and Pacific Regional Office; 2011.

\section{Submit your next manuscript to BioMed Central and take full advantage of:}

- Convenient online submission

- Thorough peer review

- No space constraints or color figure charges

- Immediate publication on acceptance

- Inclusion in PubMed, CAS, Scopus and Google Scholar

- Research which is freely available for redistribution 